\title{
Emergence of Lagrangian Field Theory from Self-Organized Criticality
}

\author{
Ervin Goldfain \\ Research Scholar, Ronin Institute, Montclair, New Jersey 07043 \\ Email: ervin.goldfain@ronininstitute.org
}

\begin{abstract}
Self-organized criticality (SOC) is a universal mechanism for self-sustained critical behavior in large-scale systems evolving outside equilibrium. Our report explores a tentative link between SOC and Lagrangian field theory, with the long-term goal of bridging the gap between complex dynamics and the nonperturbative behavior of quantum fields.
\end{abstract}

Key words: Self-organized criticality, multifractals, Lagrangian field theory, non-equilibrium dynamics, complexity theory.

\section{Key concepts of Self-Organized Criticality}

The study of equilibrium critical phenomena reveals that, near a second-order phase transition, the scaling behavior of physical observables follows the so-called finite-size scaling (FSS) ansatz. By analogy, the probability distribution defining the FSS ansatz in SOC takes the form $[1,5-6,9-10,13]$

$$
\begin{gathered}
P(s, L) \sim s^{-\tau_{s}} \Phi\left(s / s_{c}\right) \text { for } s>>1, L>>1 \\
s_{c}(L) \sim L^{D_{s}} \text { for } L>>1 \\
\mathbf{1} \mid \mathrm{P} \text { a g e }
\end{gathered}
$$


in which $s_{c}$ is the cutoff in the avalanche-size and where $\tau_{s}$ and $D_{S}$ are called the avalanche-size exponent and the avalanche dimension, respectively. Quite generally, (1) shows that, for a system of finite extent and large avalanches, the avalanche-size probability behaves as a power-law weighted by a cutoff function. By analogy with equilibrium critical phenomena, the couple of exponents $\tau_{s}$ and $D_{S}$ determine the universality class of the SOC model.

To enable all moments of (1) to exist, the cutoff function must decay sufficiently fast. One obtains the following representation of the cutoff function upon power expanding it around zero [5],

$$
\Phi(x) \sim\left\{\begin{array}{cc}
\Phi(0)+\Phi^{\prime}(0) x+\frac{1}{2} \Phi^{\prime \prime}(0) x^{2}+\ldots, & x<1 \\
\rightarrow 0, & x>1
\end{array}\right.
$$

The avalanche-size probability must be normalized to unity and its average be diverging along with $L \rightarrow \infty$, which leads to the following constraints

$$
\begin{aligned}
& \sum_{s=1}^{\infty} P(s ; L)=1 \quad \text { for } L<\infty, \\
& \langle s\rangle=\sum_{s=1}^{\infty} s P(s ; L) \rightarrow \infty \text { for } L \rightarrow \infty
\end{aligned}
$$

Under the assumption that $\Phi(0) \neq 0$, the behavior of (1) for an infinite system size may be approximated as

$$
\lim _{L \rightarrow \infty} P(s ; L) \sim s^{-\tau_{s}} \Phi(0)
$$


Furthermore, to comply with (3) and (4), the avalanche-size exponent must fall in the range

$$
1<\tau_{s} \leq 2
$$

One may transition from the framework of equilibrium critical phenomena to SOC under the plausible assumption that the correlation length $\xi$ displays the same behavior as the avalanche-size, i.e.,

$$
s=\xi ; \quad s_{c}=L
$$

\section{From SOC to the minimal fractal manifold (MFM)}

Refs. [11-12, 14, 19-22] have discussed at length the physical significance of the minimal fractal manifold (MFM), a spacetime continuum characterized by arbitrarily small and scale-dependent deviations from four dimensions $(\varepsilon=4-D<<1)$. The MFM reflects an evolving setting that starts far-from-equilibrium and gradually reaches the equilibrium conditions mandated by field theory in the limit of four-dimensional spacetime $(\varepsilon=0)$. There are well-motivated reasons to believe that dimensional fluctuations driven by $\varepsilon$ are asymptotically compatible with the internal structure and dynamics of the Standard Model of particle physics [11-12, 14, 19-22].

Based on these premises, as well as on (7), we introduce the hypothesis that the dimensional deviation $\varepsilon$ and the avalanche-size $s=\xi$ are interchangeable concepts via

$$
\varepsilon=4-D=s^{-1}<<1
$$


This hypothesis is consistent with the philosophy of conformal field theory, as the fourdimensional limit $\varepsilon=0,(D=4)$ naturally matches the asymptotic approach to the farinfrared regime of massless fields $\left(m=\xi^{-1}=s^{-1} \rightarrow 0\right)$.

Furthermore, since $\varepsilon$ is conjectured to flow with the energy scale, it likely reaches its uppermost observable value close to the formation of the cosmic microwave background (CMB) [16]. The maximal dimensional deviation is therefore set to

$$
\varepsilon_{c}=\varepsilon_{\max } \approx 10^{-5} ; \varepsilon<<\varepsilon_{c}
$$

which turns (1) into

$$
\begin{gathered}
P\left(\varepsilon, \varepsilon_{c}\right) \sim \varepsilon^{\tau_{s}} \Phi\left(\varepsilon_{c} / \varepsilon\right), \varepsilon<<1 \\
\varepsilon_{c}(\mu) \sim \mu^{D_{s}}, \mu \gg>1
\end{gathered}
$$

where $\mu$ is the dimensionless Renormalization Group scale.

It can be shown that, in general, the FSS ansatz (1) or (10) can be characterized through a set of critical exponents $\alpha$ defined through [2, 9]

$$
\varepsilon_{c}^{f(\alpha)}=\int_{\varepsilon_{c}^{\alpha}}^{\infty} P\left(\varepsilon, \varepsilon_{c}\right) d \varepsilon
$$

or,

$$
f(\alpha)=\frac{\log \int_{\varepsilon_{c}^{\alpha}}^{\infty} P\left(\varepsilon, \varepsilon_{c}\right) d \varepsilon}{\log \varepsilon_{c}}
$$


The real numbers $\alpha$ represent the so-called Lipschitz-Hölder (LH) exponents and the multifractal spectrum $f(\alpha)$ quantifies their continuous distribution in the range $\alpha \in\left[\alpha_{\min }, \alpha_{\max }\right]$. As the two next sections show, the multifractal spectrum plays a crucial role in bridging the gap between SOC and Lagrangian field theory.

\section{Key concepts of multifractal analysis}

Since multifractals and SOC are closely related, we now take a brief detour to delve into the topic of multifractal analysis.

From a mathematical standpoint, multifractal analysis is a theory of self-similar measures [2]. A measure is defined as function that assigns a number to certain subsets of a given set: the number is said to represent the measure of the set. The basic properties of measures are extensions of the geometrical concepts of length, area and volume, so that - for example - the measure of the union of two disjoint sets is the sum of the measures of the two sets, and the measure of the empty set is zero. Roughly speaking, a self-similar measure is a measure whose geometrical attributes stay unchanged upon arbitrary scaling operations.

Following [2-3] in detail, let a set $\Sigma$ supporting a measure be covered with a collection of boxes of size $\varepsilon=4-D<<1$. The number of boxes needed to cover the set is defined through the scaling

$$
N(\varepsilon) \sim \varepsilon^{-D_{H}}
$$


in which $D_{H}$ is the Hausdorff dimension of the set, which is adequate for characterization of mono-fractals. In general, the quantitative description of multifractal measures requires replacing $D_{H}$ with a continuous LH exponent $\alpha$ according to

$$
\mu \sim \varepsilon^{\alpha}, \quad 0<\alpha \in\left[\alpha_{\min }, \alpha_{\max }\right]<\infty
$$

The number of boxes of size $\varepsilon$ having the LH exponent $\alpha$ is given by

$$
N_{\varepsilon}(\alpha) \sim \varepsilon^{-f(\alpha)}
$$

where the distribution of LH exponents follows the multifractal spectrum $f(\alpha)$. The meaning of (15) is that there are infinitely many subsets of boxes having the LH exponent $\alpha$ in the limit $\varepsilon \rightarrow 0$.

By analogy with equilibrium statistical mechanics and Quantum Field Theory, multifractal analysis is based on a partition function defined as

$$
Z_{q}(\varepsilon)=\sum_{i=1}^{N(\varepsilon)} \mu_{i}^{q}, q \in \mathbf{R}
$$

By (14), the measures assigned to boxes $i=1,2, \ldots, N(\varepsilon)$ is $\mu_{i}=\varepsilon^{\alpha_{i}}$. Assuming that the number of boxes for which $\alpha<\alpha_{i}<\alpha+d \alpha$ is $N_{\varepsilon}(\alpha) d \alpha$, the contribution of the subset of boxes with $\alpha_{i} \in[\alpha, \alpha+d \alpha]$ to the partition function is $N_{\varepsilon}(\alpha)\left(\varepsilon^{\alpha}\right)^{q} d \alpha$ and thus

$$
Z_{q}(\varepsilon)=\int N_{\varepsilon}(\alpha)\left(\varepsilon^{\alpha}\right)^{q} d \alpha
$$

By (15) and (17), we obtain

$$
6 \text { | P a g e }
$$




$$
Z_{q}(\varepsilon)=\int \varepsilon^{q \alpha-f(\alpha)} d \alpha
$$

In the limit of four-dimensional spacetime $\varepsilon \rightarrow 0(D=4)$, the prevailing contribution to the integral (18) arises from those values of the exponent $\alpha$ that minimize the sum $q \alpha-f(\alpha)[2]$. Such minimum exists if

$$
\frac{\partial}{\partial \alpha}\{q \alpha-f(\alpha)\}=0
$$

which implies two conditions, namely

$$
q=\frac{\partial}{\partial \alpha} f(\alpha), \quad \alpha=\alpha(q)
$$

and

$$
\frac{\partial^{2}}{\partial \alpha^{2}} f(\alpha)<0, \quad \alpha=\alpha(q)
$$

Introducing the definition

$$
\tau(q)=q \alpha(q)-f(\alpha(q))
$$

it can be shown that

$$
\alpha(q)=\frac{\partial}{\partial q}\left[\tau(q)+K_{1}\right]
$$


in which $K_{1}$ represents a constant independent of $q$, that is, $\partial K_{1} / \partial q=0$. By (20), relation (22) can be presented as

$$
\alpha(q)=\frac{\tau(q)}{q}+f(\alpha(q)) \frac{\partial\left[\alpha(q)+K_{2}\right]}{\partial f(\alpha(q))}
$$

where $K_{2}$ is a constant independent of the multifractal spectrum, that is, $\partial K_{2} / \partial f=0$. Equations (22) (or (24)) and (23) give a parametric representation of the spectrum $f(\alpha)$ in terms of $q$. Taken together, (22) and (23) act as a Legendre transform from the variables $q$ and $\tau$ to the variables $\alpha$ and $f$.

The Legendre transform is frequently used in various areas of theoretical physics including classical mechanics, statistical mechanics and thermodynamics, as well as Quantum Field Theory (QFT) [15]. Next section sets up the connection between (24), on the one hand, and the Legendre transform of Lagrangian field theory, on the other.

\section{From Multifractals to Lagrangian dynamics}

Starting from (24), it can be shown that classical statistical mechanics offers a straightforward analog of multifractal analysis. With reference to Tab. 1, the temperature $(T)$, internal energy $(U)$, entropy $(S)$ and free energy $(F)$ are respectively echoed in multifractal theory by $q^{-1}, \alpha, f(\alpha)$ and $\tau(q) / q[2,9]$. Considering this analogy, the thermodynamic equation

$$
F(T, V)=U(S, V)-T S
$$

turns into a replica of the Legendre transform (24), where $V$ stands for volume and

$$
8 \text { | P a g e }
$$




$$
d U=T d S-p d V \Rightarrow T=\left(\frac{\partial U}{\partial S}\right)_{V}
$$

\begin{tabular}{|c|c|}
\hline Multifractals & Statistical Mechanics \\
\hline$q$ & $1 / T$ \\
\hline$\alpha(q)$ & $U$ \\
\hline$\tau(q) / q$ & $F$ \\
\hline$f(\alpha)$ & $S$ \\
\hline
\end{tabular}

Tab 1: Mapping Multifractals to Statistical Mechanics

( $T$ = temperature, $U=$ energy, $F=$ free energy, $S=$ entropy)

It is well known that the dynamics of generic classical fields is governed by the least-action principle $[8,17]$

$$
S=\int d^{4} x L\left(\varphi, \partial_{\mu} \varphi\right)
$$

where $S$ is the action whose minimization yields the Euler-Lagrange equations

$$
\delta S=0 \Rightarrow \partial_{\mu} \frac{\delta L}{\delta\left(\partial_{\mu} \varphi\right)}-\frac{\delta L}{\delta \varphi}=0
$$

The Hamiltonian associated with (27) is defined by

$$
H(x)=\pi(x) \partial_{0} \varphi(x)-L(x)
$$

in which the conjugate momentum is 


$$
\pi(x)=\frac{\delta L}{\delta\left(\partial_{0} \varphi\right)}
$$

One can interpolate between Lagrangian and Hamiltonian formulation of the theory using the kinetic $(T)$ and potential $(V)$ components according to

$$
L=T-V, H=T+V \Rightarrow L=H-2 V
$$

By (31), if the potential is independent of $\partial_{0} \varphi$, (30) can be written as

$$
\pi(x)=\frac{\delta L}{\delta\left(\partial_{0} \varphi\right)}=\frac{\delta H}{\delta\left(\partial_{0} \varphi\right)}, \text { if } \frac{\delta V}{\delta\left(\partial_{0} \varphi\right)}=0
$$

Inspection of the first entry in Tab. 1 hints to the analogy

$$
q \Leftrightarrow\left|t_{E}\right|=1 / T
$$

where $t_{E}=-i x_{0}$ is interpreted as Euclidean time, identical with the inverse temperature of statistical mechanics expressed in natural units $(k=1)$ [18]. Side by side evaluation of (29) to (32) with (22) to (24) leads to the following operational identification

$$
\begin{gathered}
\partial_{0} \Leftrightarrow \partial_{t_{E}} \\
\alpha(q) \Leftrightarrow H \\
-\frac{\tau(q)}{q} \Leftrightarrow L \\
\partial_{0} \varphi \Leftrightarrow f(\alpha) \\
\mathbf{1 0} \mid \mathrm{P} \text { a g e }
\end{gathered}
$$




$$
\begin{gathered}
\varphi \Leftrightarrow \varphi(\alpha)=\int f(\alpha) d\left|t_{E}\right| \approx\langle f(\alpha)\rangle \cdot\left|t_{E}\right| \\
K_{1}=K_{2} \Leftrightarrow-2 V
\end{gathered}
$$

Therefore $\varphi(\alpha)$ and $f(\alpha)$ form a pair of conjugate variables defining the Lagrangian field analog of SOC. Relations (33)-(37) are consolidated in Tab. 2.

\begin{tabular}{|c|c|}
\hline Multifractals & Lagrangian dynamics \\
\hline$q$ & $\left|t_{E}\right|$ \\
\hline$\alpha(q)$ & $H$ \\
\hline$\tau(q) / q$ & $-L$ \\
\hline$f(\alpha)$ & $\partial_{0} \varphi$ \\
\hline
\end{tabular}

Tab 2: Mapping Multifractals to Lagrangian dynamics

$$
\text { ( } \left.t_{E}=\text { Euclidean time, } H=\text { Hamiltonian, } L=\text { Lagrangian }\right)
$$

A key observation is now in order. Integrating (36) over the Euclidean time (33) shows that

$$
S=-\int\left(\tau\left(\left|t_{E}\right|\right) /\left|t_{E}\right|\right) d^{3} x d\left|t_{E}\right| \approx-\left\langle\tau\left(\left|t_{E}\right|\right) /\left|t_{E}\right|\right) \cdot \Delta^{3} \cdot\left|t_{E}\right|
$$

in which $\Delta$ denotes the spatial domain of integration. Taken together, (19), (28) and (40) hint that multifractal geometry and SOC lie at the root of the least-action principle.

We close by noting that the sole intent of our work is to shed light on the tantalizing connection between Lagrangian dynamics and SOC. Future research may focus on several aspects of field theory that were not covered here, such for example, the propagator 
structure of QFT and the FSS ansatz (1) [7]. The reader is also directed to [14] for a preliminary analysis of the relationship between the Legendre transform and the path integral formalism of perturbative QFT.

\section{$\underline{\text { APPENDIX }}$}

A closer inspection of (15) reveals that an arbitrary change of $f(\alpha)$ is formally equivalent to a shift in $\varepsilon=4-D<<1$, in response to a change of the dimensionless RG scale $\mu$ [23]. To derive the connection between the two scaling relationships, we require the following alternative definition of (15) to hold true

$$
N_{\varepsilon}(\alpha)=\varepsilon\left(\mu_{0}\right)^{-f(\alpha)}=\varepsilon(\mu)^{-f\left(\alpha_{0}\right)}
$$

where $\mu_{0}$ and $\alpha_{0}$ denote two reference values. (A1) leads to

$$
\varepsilon(\mu)=\varepsilon\left(\mu_{0}\right)^{\sigma\left(\alpha, \alpha_{0}\right)}
$$

in which

$$
\sigma\left(\alpha, \alpha_{0}\right)=\frac{f(\alpha)}{f\left(\alpha_{0}\right)}
$$

Consider next the simple case of a free particle of mass $m_{0}$ moving at low speed with linear momentum $p$ in one space dimension. The dispersion relationship linking the linear momentum with energy is 


$$
m_{0}=\frac{p^{2}}{2 E}
$$

Passing to the Euclidean representation $E_{E}=-i E$ in (A4) yields the Euclidean mass $m_{0, E}$ in the form

$$
m_{0, E}=-\frac{i p^{2}}{2 E_{E}}
$$

When translated in terms of (35) and (37), the above equation suggests that the counterpart of Euclidean mass in the SOC framework is given by

$$
\left|m_{0, E}\right|=\frac{f^{2}(\alpha)}{2 \alpha}
$$

(A6) represents a cubic function in $\alpha$, if the spectrum $f(\alpha)$ is assumed to be quadratic. (A2) and (A6) hint that the dynamical property of "mass" emerges from the continuous dimensionality of spacetime $\varepsilon=4-D<<1$, an argument made throughout our previous publications (see for example [11-12]).

\section{References:}

1. https://arxiv.org/pdf/cond-mat/9805045.pdf

2. Evertsz, C. J. G and Mandelbrot, B. "Multifractal Measures", in "Chaos and Fractals, New Frontiers of Science", Springer-Verlag (1992).

3. https://www.sciencedirect.com/science/article/abs/pii/o920563287900363

4. https://arxiv.org/pdf/1606.02957.pdf 
5. Christensen, K. and Moloney, N. R., "Complexity and Criticality", Imperial College Press 2005 .

6. Available at the following site: http://www.smmlab.it/zapperi/reprints/Vespignani Physical\%2oReviewE57 634519 98.pdf

7. Available at the following site:

https://www.researchgate.net/publication/340514250 Quantum Field Theory as M anifestation of Self-Organized Criticality

8. http://www.scholarpedia.org/article/Lagrangian formalism for fields

9. https://arxiv.org/pdf/cond-mat/9903270.pdf

10. https://arxiv.org/pdf/cond-mat/9811365.pdf

11. Available at the following sites:

http://www.aracneeditrice.it/aracneweb/index.php/pubblicazione.html?item=9788854 $\underline{889972}$

https://www.researchgate.net/publication/278849474_Introduction_to_Fractional_Fi eld Theory consolidated_version

12. Available at the following site:

https://www.researchgate.net/publication/228625050 Chaotic Dynamics of the Re normalization Group Flow and Standard Model Parameters Intl

13. https://arxiv.org/pdf/1310.5527.pdf

14. Available at the following site:

https://www.academia.edu/38752380/Multifractal Geometry and Standard Model Symmetries 
15. https://arxiv.org/pdf/1612.00462.pdf

16. https://arxiv.org/pdf/0806.2675.pdf

17. Cheng, T. P, and Li L.F., "Gauge Theory of Elementary Particle Physics", Clarendon Press, Oxford, 1989.

18. http://maths.dur.ac.uk/users/kasper.peeters/pdf/eft.pdf

19. https://www.prespacetime.com/index.php/pst/article/view/1619/1546

20. https://www.prespacetime.com/index.php/pst/article/view/1653/1575

21. https://www.prespacetime.com/index.php/pst/article/view/1648/1569

22. https://www.prespacetime.com/index.php/pst/article/view/1656/1570

23. https://arxiv.org/pdf/hep-th/o310213.pdf 